\title{
On the Plastic Deformation of Pure Solid Lithium Sulfate and Lithium-Potassium Sulfate Mixtures
}

\author{
Bengt Augustsson and Arnold Lundén \\ Department of Physics, Chalmers University of Technology, Göteborg, Sweden \\ (Z. Naturforschg. 21 a, 1860-1863 [1966] ; received 23 August 1966)
}

\begin{abstract}
A simple rotational viscometer has been constructed for measurements at elevated temperatures in the region $10^{9}-2 \times 10^{12}$ poise, and has been used to study the deformation of pure fcc lithium sulfate (temperature range $740-860^{\circ} \mathrm{C}$ ) and mixtures of this salt with potassium sulfate. These substances resemble highly-viscous non-Newtovian liquids, and for a given torque the apparent viscosity can be represented by the conventional relation $\eta=\eta_{0} \exp (Q / R T)$. For pure lithium sulfate, measurements with a torque of $19 \times 10^{7}$ dyne-cm gave the values $\eta_{0}=4.24 \times 10^{-4}$ poise and $Q=7.4 \times 10^{4} \mathrm{cal} / \mathrm{mole}$ for the two constants.

Two mixtures containing 1.0 and 2.2 mole- $\% \mathrm{~K}_{2} \mathrm{SO}_{4}$, respectively, were studied. In the homogeneous solid solution phase the viscosity showed a dependence on the thermal prehistory of the salt during the first few days at a given temperature. This effect was not detected for the pure lithium sulfate or for the two-phase region of the mixtures. The temperature dependence of the viscosity was less pronounced for the homogeneous phase than it was for the pure salt, while it was very great in the two-phase region. It is suggested that viscosity (or electrical conductivity) measurements should be a possible method to determine the position of the solidus curve for mixtures of this type.
\end{abstract}

It has recently been demonstrated that the addition of $\mathrm{K}_{2} \mathrm{SO}_{4}$ to $\mathrm{Li}_{2} \mathrm{SO}_{4}$ causes a considerable change in the rheological properties of the cubic (fcc) high-temperature modification ${ }^{1}$. The rate with which a sphere of stainless steel penetrated through the salt was studied in this first investigation. In order to obtain more accurate information on the deformation of high temperature modifications of salts, a simple rotational viscometer was constructed, see Figs. 1 and 2.

The inner cylinder can be removed for the filling procedure. About 250 grams of salt is added as lumps, melted and then allowed to solidify. The torque is applied by letting two $5 \mathrm{~kg}$ weights pull a horizontal disc, radius $200 \mathrm{~mm}$. The angular velocity $(\omega)$ of the disc is measured by means of a lamp, mirror and scale. If the shearing stress is assumed to be proportional to the velocity gradient, the viscosity $(\eta)$ of the investigated material can be calculated from the following expression for the external torque

$$
\begin{aligned}
M_{0}= & 4 \pi \eta \omega L R_{1}{ }^{2} R_{2}{ }^{2}\left(R_{2}{ }^{2}-R_{1}{ }^{2}\right)^{-1} \\
& +\frac{1}{2} \pi \eta \omega R_{1}{ }^{4} d^{-1}+M_{L}
\end{aligned}
$$

with $L=$ height of the salt cylinder, $R_{1}=$ inner, $R_{2}$ = outer radius, and $d=$ distance between bottom

1 A. Lundén, B. Jonson, and B. Augustsson, Z. Naturforschg. 21 a, 593 [1966]; on p. 594, col. 2, line 4 read "radius $1.0 \mathrm{~cm}$ ".

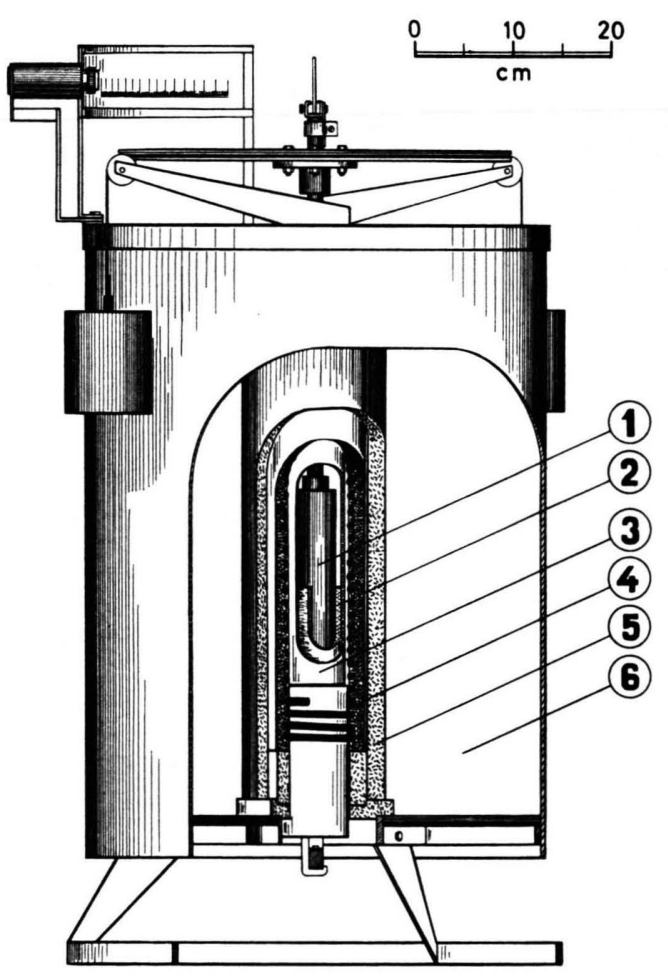

Fig. 1. Viscometer for measurements at elevated temperature: 1. Rotating cylinder, 2. salt, 3 . fixed cylinder, 4 . heating coil, 5 . ceramic tube, 6 . insulation.

surfaces of the two cylinders. The last term, $M_{L}$, corresponds to the contributions from edge effects and from friction in the bearings. For our apparatus 


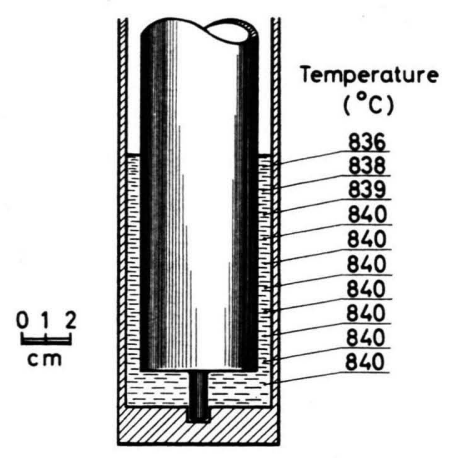

Fig. 2. Temperature distribution in the salt.

we have $R_{1}=22.0 \mathrm{~mm}, R_{2}=29.5 \mathrm{~mm}$, and $d=15$ mm. $L=90 \mathrm{~mm}$ is a typical value. We have estimated that $M_{L} \cong 10 \eta \omega$. We thus obtain typically

$$
M_{0}=\eta \omega(1230+25+10) \text { dyne-cm. }
$$

The heating coil was supplied from an AC-voltage stabilizer. A thermocouple was placed at the bottom of the inner cylinder. By using an adjustable standard voltage source together with a microvoltmeter as null detector, the temperature was measured with an accuracy of about $0.2 \mathrm{degr}$. Since the change of viscosity at the melting point of pure $\mathrm{Li}_{2} \mathrm{SO}_{4}$ is very sharp, the thermocouple could be calibrated relative to this temperature, taken as $860^{\circ} \mathrm{C}$. The temperature fluctuation during a measurement was less than 0.25 degr. per hour. Since the salt layer is rather thin (ca. $7 \mathrm{~mm}$ ), radial temperature gradients are negligible. In the vertical direction there is, however, a slight temperature gradient just below the salt surface ${ }^{2}$, cf. Fig. 2.

The two cylinders are made of stainless steel. There were no signs of corrosion of the surfaces that were in contact with the salt, even for periods of several months.

a- $\mathrm{Li}_{2} \mathrm{SO}_{4}$ has a fcc structure in which $\mathrm{K}_{2} \mathrm{SO}_{4}$ is soluble to a certain extent, forming an isomorphous phase ${ }^{3}$. The solidus curve of the phase diagram has not been determined experimentally, while the liquidus curve has been studied by several investigators (l. c. $^{3-5}$ ), with considerable deviations between the results.

2 The temperature distribution was measured with a thermocouple that was moved inside a thin steel tube that had been placed in the salt between the two cylinders. Heat conductivity along the steel tube might introduce a slight error in the measurements near the salt surface, i. e. the actual vertical temperature difference within the salt might
Special attention has been paid to the reproducibility of the viscosity measurements, since we had found earlier, that the thermal pretreatment of the salt was of importance for the results ${ }^{1}$.

In order to get information on existing hysteresis and relaxation phenomena we chose to select the temperatures of consecutive measurements arbitrarily over a large range. (The limits of the temperature interval within which measurements were practical with our apparatus, were exceeded deliberately between some of the measurements, too.) After the current had been adjusted, it took up to about 16 hours until the temperature was constant. For each measurement the angular velocity was registered over some eight hours.

\section{Results and Discussion}

The present investigation is restricted to the measuring range of the viscometer, approximately $10^{9}$ to $2 \times 10^{12}$ poise. A preliminary investigation confirmed that, at a given temperature, the angular velocity is not proportional to the applied torque, i. e. the substance has a non-Newtovian character. However, it is possible to measure apparent viscosities for chosen torques. (All the results reported here are for a torque of $19 \times 10^{7}$ dyne-cm.) We decided to study pure $\mathrm{Li}_{2} \mathrm{SO}_{4}$ and two mixtures, one of these mainly in the homogeneous, the other mainly in the two-phase region. Neither for the pure salt nor for the two-phase region could we detect any influence of the thermal pretreatment. On the other hand, for the homogeneous region the viscosity showed a dependence upon time the first hours after the temperature had stabilized. This effect is demonstrated in Table 1, showing the results of experiments where the system first was kept at a certain temperature for about 3 days, whereupon the temperature was changed, and the viscosity was measured during three days. Several checks of this type were made, and, in general, the time-dependence was more pronounced when the temperature was increased than when it was decreased. Only the measurements done

be smaller during a viscosity measurement than indicated in Fig. 2.

3 R. Nacken, Neues Jahr. Mineral. Geol., Beilage Bd. 24 A, 43 [1907].

${ }^{4}$ O. S. Dombrowskaja, Zh. Obshch. Khim. 3, 1007 [1933].

5 A. G. Bergman, A. I. Kislova, and V. I. Posypaiko, Dokl. Akad. Nauk SSSR 88, 815 [1953]. 


\begin{tabular}{|c|c|c|}
\hline $\begin{array}{c}\text { Temperature } \\
{ }^{\circ} \mathrm{C}\end{array}$ & $\begin{array}{c}\text { Time } \\
\text { hours }\end{array}$ & $\begin{array}{c}\text { Viscosity } \\
\text { TP }\end{array}$ \\
\hline 690 & 0 & - \\
754 & 21 & 0.468 \\
755 & 45 & 0.433 \\
755 & 70 & 0.430 \\
\hline 755 & 0 & - \\
694 & 23 & 2.68 \\
694 & 46 & 2.92 \\
695 & 70 & 2.94 \\
\hline
\end{tabular}

Table 1. Time dependence of the viscosity in the homogeneous phase. The mixture contained 1.0 mole- $\% \mathrm{~K}_{2} \mathrm{SO}_{4}$. Each measurement took 8 hours. The time is quoted for the middle of the interval. $1 \mathrm{TP}=10^{12}$ poise.

after sufficient time had elapsed were included, when the results were plotted in Fig. 3.

For the pure salt there was a sharp change in viscosity at the melting point, $860{ }^{\circ} \mathrm{C}$, while for the mixtures the transition was diffuse. The intersection with the solidus curve was at about $770{ }^{\circ} \mathrm{C}$ for a mixture with 1.0 mole- $\% \mathrm{~K}_{2} \mathrm{SO}_{4}$, and at about $675{ }^{\circ} \mathrm{C}$ for 2.2 mole- $\%$.
Experimental results on transport properties are frequently expressed by exponential relations ${ }^{6}$ :

$$
\eta=\eta_{0} \exp (B / T)
$$

where $T$ is the absolute temperature. The entity $Q=R B$ is called the activation energy of the process ( $R$ is the gas constant), see Table 2 . The error quoted for $Q$ is at the $95-\%$ confidence level ${ }^{7}$. Due

\begin{tabular}{|c|c|c|}
\hline $\begin{array}{c}\text { Mole-\% } \\
\mathrm{K}_{2} \mathrm{SO}_{4}\end{array}$ & $\begin{array}{c}\text { Temp.range } \\
{ }^{\circ} \mathrm{C}\end{array}$ & $\begin{array}{c}Q=R \cdot B \\
\text { cal } / \text { mole }\end{array}$ \\
\hline 0 & $740-860$ & $(7.4 \pm 1.5) 10^{4}$ \\
1.0 & $670-765$ & $(5.2 \pm 0.3) 10^{4}$ \\
2.2 & $675-740$ & $(13.8 \pm 0.8) 10^{4}$ \\
\hline
\end{tabular}

Table 2. Temperature dependence of viscosity. Errors of $Q$ are quoted at the $95 \%$ confidence level.

to the limits of the measuring range of the present device, the results for the $2.2 \%$ mixture in the homogeneous phase (as well as those for the $1.0 \%$ mixture in the two-phase region) are rather uncertain, but

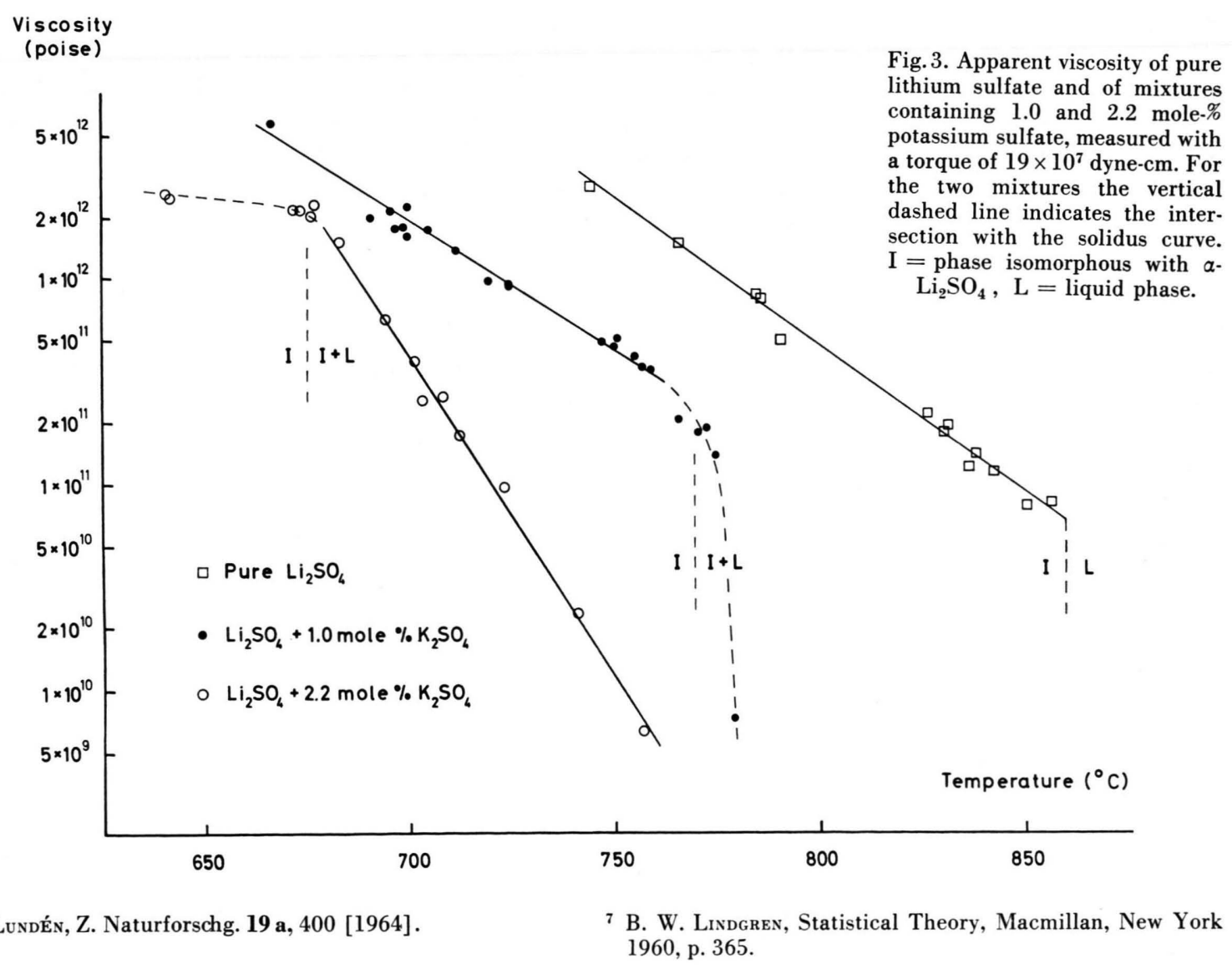

${ }^{6}$ A. LundÉn, Z. Naturforschg. 19 a, 400 [1964]. B. W. LINDG
1960, p. 365. 
it is possible to estimate that $Q$ is less than about $5 \times 10^{4} \mathrm{cal} / \mathrm{mole}$ in this phase.

Although the physical meaning of the concept of activation energy is not quite clear in all cases ${ }^{6}$, it is tempting to use this entity when discussing the results. For pure $\mathrm{Li}_{2} \mathrm{SO}_{4}$ the calculated activation energy of viscosity is an order of magnitude higher than the activation energies of conductivity (8.3 $\times 10^{3} \mathrm{cal} /$ eq. $)$ and cation self-diffusion ${ }^{8}$. A difference like this should be expected ${ }^{9}$, since a larger energy should be required for a process that involves the deformation of the sulfate lattice than for merely transporting the mobile cations. Following this argument, the lattice might be easier to deform when it is partly distorted by the presence of large impurity cations, and we indeed find for the homogeneous phase that $Q$ is the lower the more $\mathrm{K}_{2} \mathrm{SO}_{4}$ has been added. In the two-phase region the large change of viscosity with temperature obviously is due to the change in the proportions of the solid and liquid fractions.

Kvist has recently studied the electrical conductivity of solid $\mathrm{Li}_{2} \mathrm{SO}_{4}-\mathrm{K}_{2} \mathrm{SO}_{4}$ mixtures ${ }^{10}$. For the two-phase region, as well as for pure $\mathrm{Li}_{2} \mathrm{SO}_{4}$, the

8 A. Kvist and A. LundÉn, Z. Naturforschg. 20 a, 235 [1965].

9 A factor of two might be due to the fact that the activation energies of conductivity and cation diffusion refer to an equivalent while $Q$ for viscosity corresponds to a mole of salt. conductivity is the same whether measured with increasing or decreasing temperature, but for the homogeneous phase there is a branching in two curves depending on the direction in which the temperature is changed. Similar phenomena are thus observed for viscosity and for electrical conductivity, and measurements of either of these properties might serve as a method to determine the position of the solidus curve.

At a given temperature the viscosity is an order of magnitude lower for the mixture with $1 \% \mathrm{~K}_{2} \mathrm{SO}_{4}$ than for pure $\mathrm{Li}_{2} \mathrm{SO}_{4}$, i. e. the presence of impurity cations facilitates the deformation of the salt. This observation might be explained in alternative ways, depending on the model that is chosen for the system. However, at the present stage of the experiments, all interpretations would be very speculative. Our present work emphasizes liquid-like properties, and supplementary investigations are needed before a deeper explanation of the transport properties can be given.

This work is supported by the Swedish Technical Research Council.

10 A. Kvist, Z. Naturforschg. 21 a, 1221 [1966]. 